\title{
FICHA DE REGISTRO ODONTOLÓGICO CON FINES FORENSES
}

\section{DENTAL RECORD FORM FOR FORENSIC PURPOSES}

\author{
Marcelo David Mancheno Dávila ${ }^{1}$ \\ MSc. Verónica Paulina Cáceres Manzano² \\ Ph.D. María Eugenia LUCENA ${ }^{3}$ \\ Ph.D. Luisa Carolina González Ramírez ${ }^{4}$ \\ Universidad Nacional de Chimborazo \\ Méd, María de los Ángeles Galarza Pazmiño ${ }^{5}$ \\ Méd Esp, Jorge Marcelo Quintana Yánez ${ }^{6}$
}

Servicio Nacional de Medicina

Ecuador

1 Marcelo David Mancheno ORCID: https://orcid.org/0000-0001-6152-0868

Universidad Nacional de Chimborazo, Estudiante, Facultad de Ciencias de la Salud, km 3/1/2 Vía Guano Campus Edison Rivera, Riobamba.manchenomarcelo4@gmail.com

2 MSc. Verónica Paulina Cáceres M. ORCID: https://orcid.org/0000-0001-9560-9625

vcaceres@unach.edu.ec Universidad Nacional de Chimborazo, Estudiante, Facultad de Ciencias de la Salud, km 3/1/2 Vía Guano Campus Edison Rivera, Riobamba.

3 Ph.D. María Eugenia Lucena - ORCID: https://orcid.org/0000-0001-9120-345X

Universidad Nacional de Chimborazo, Grupo de Investigación "Análisis de Muestras Biológicas y Forenses". Universidad Nacional de Chimborazo, Docente Facultad de Ciencias de la Salud, km 3/1/2 Vía Guano Campus Edison Rivera, Riobamba. mlucena@unach.edu.ec

4 Ph.D. Luisa Carolina González Ramírez Universidad Nacional de Chimborazo, https://orcid.org/0000-0002-4431-965X Universidad Nacional de Chimborazo, Grupo de Investigación "Análisis de Muestras Biológicas y Forenses" Docente Facultad de Ciencias de la Salud, km 3/1/2 Vía Guano Campus Edison Rivera, Riobamba. Icgonzalez@unach.edu.ec

$5 \quad$ Méd, María de los Ángeles Galarza Pazmiño.ORCID:https://orcid.org/0000-0003-4001-3458

Servicio Nacional de Medicina Legal y Ciencias Forenses, Parque Industrial calle F. angelesgalarzapazmino@gmail.com

$6 \quad$ Méd Esp, Jorge Marcelo Quintana Yánez, ORCID: https://orcid.org/0000-0001- 7918-556X

Servicio Nacional de Medicina Legal y Ciencias Forenses, Parque Industrial calle F. jorge.quitana@cienciasforenses.gob.ec 


\section{RESUMEN}

La presente investigación tuvo como objetivo desarrollar una ficha de registro odontológico para su uso en investigaciones forenses. Se realizó un estudio de tipo exploratorio, descriptivo y correlacional, de corte transversal, no experimental y de campo. Como técnicas de recolección de datos se aplicó la observación y sus instrumentos fueron la lista de cotejo y las historias clínicas de las cuales se analizó la consistencia del Ilenado; otra técnica utilizada fue la encuesta con el cuestionario como instrumento. La población de estudio estuvo constituida por 150 estudiantes de los cuales se seleccionó una muestra de 108. Los resultados observados indicaron que la información ingresada en el historial clínico fue medianamente satisfactorios en la sección de datos intraorales $(60,2 \%)$, los datos extraorales no satisfactorio $(51,9 \%)$, el registro de exámenes complementarios no satisfactorios $(63 \%)$, pues presentaron debilidades en el llenado de todas estas secciones. A partir de los resultados obtenidos en las evaluaciones se pudo determinar características y datos adecuados para la implementación de una ficha de registro odontológico aplicable en investigaciones de tipo forense.

PALABRAS CLAVE: odontología forense, ficha de registro odontológico, identificación de personas, mantenimiento de registros.

\section{ABSTRACT}

This research aims to develop a dental record form for use in forensic investigations. An exploratory, descriptive and correlational, cross-sectional, non-experimental and field study was conducted. As data collection techniques, the observation was applied and its instruments were the checklist and the medical records of which the consistency of the filling was analyzed, other technique used with the questionnaire as an instrument was used. The study population consisted of 150 students from which a sample of 108 was chosen. Results indicate that the information register in the clinical record was moderately satisfactory in the intraoral data section $(60.2 \%)$, the extraoral data not satisfactory $(51.9 \%)$, the registration of complementary exams with unsatisfactory of $(63 \%)$ as they presented weaknesses in the filling of all these sections. In this way, on the basis of the results obtained in the evaluations, it was possible to determine characteristics and data suitable for the implementation of a dental record sheet applicable in forensic investigations.

KEYS WORDS: forensic dentistry, dental record, identification of people, record keeping.

\section{INTRODUCCIÓN}

La cavidad oral y las estructuras que la conforman se articulan en un equilibrio que de alterarse, se afectará la salud física integral, incidiendo en las esferas mental y social de una u otra manera; de ahí que, en la búsqueda constante de la devolución del equilibrio perdido, cada tratamiento individualizado en el aspecto físico constituya también un elemento de identificación: una prótesis, la cicatriz posterior a una cirugía o un implante dental. Los dientes proveen información valiosa que ayuda a identificar a una persona con los métodos propios de la odontología legal y forense ${ }^{(1)}$. La odontología forense es una rama de la odontología donde se da importancia a la aplicación de la práctica y conocimientos odontológicos para contribuir con investigaciones de identificación y en donde se involucran todos los procesos de un correcto registro y manejo de evidencias de carácter dental para una posterior valoración y la presentación apropiada de los hallazgos en interés de la justicia ${ }^{(2,3)}$.

La característica principal de la ficha de registro de pacientes con fines forenses es realizar un adecuado proceso de recopilación de datos, almacenaje y conservación de cualquier 
elemento que se haya usado para realizar un correcto diagnóstico y su plan de tratamiento, y que este sea de fácil y rápido acceso para las instituciones encargadas del proceso de investigación forense; de manera que para analizar esta problemática, es necesario mencionar que una de sus causas es la falta o inadecuado almacenaje de datos, modelos de estudio y exámenes complementarios; actualmente no existe un protocolo que permita acceder a un banco de información adecuado para procesos investigativos $y$ en muchas ocasiones el perito forense evidencia la falta de cooperación por parte del odontólogo profesional y en formación.

La investigación de esta problemática se realiza por un interés profesional, educativo e Institucional de ámbito forense, para el apoyo a investigaciones y a su vez recalcar la importancia de un correcto almacenaje y conservación de datos que puedan ser usados para resolver diferentes casos de esta especialidad, se realizó mediante la revisión de protocolos de registro de otros países con el objeto de elaborar una propuesta que se adapte a las necesidades y características de nuestra sociedad. La población de estudio está conformada por 150 estudiantes de la clínica de Odontología de la Universidad Nacional de Chimborazo, con una muestra 108 estudiantes que fueron evaluados mediante una encuesta y un cuestionario como instrumentos de recolección de datos; el proceso de muestreo fue determinado mediante una fórmula en función de la variables de estudio.

La presente investigación está enfocada hacia la práctica forense con la propuesta de una ficha de registro odontológico para pacientes que acuden a la clínica odontológica de la Universidad Nacional de Chimborazo, la cual puede contribuir en investigaciones a través del reconocimiento de la cavidad oral.

\section{ODONTOLOGÍA FORENSE}

\section{METODOLOGÍA}

La investigación tiene un nivel de alcance descriptivo y correlacional. Con diseño de campo no experimental, enfoque mixto, de corte transversal.

\section{POBLACIÓN DE ESTUDIO}

La población estuvo constituida por 150 estudiantes de la Universidad Nacional de Chimborazo, que estaban realizando sus prácticas profesionales en la Clínica Odontológica de la UNACH.

\section{MUESTRA}

A una población de 150 estudiantes, se aplicó la fórmula de cálculo de la muestra con un índice de probabilidad del $95 \%$ y un error del 5\%. Dando como resultado una muestra de 108 estudiantes.

$$
\mathrm{n}=\frac{N^{*}\left(\alpha_{c} * 0,5\right)^{2}}{1+\left(e^{2} *(N-1)\right.}
$$

Dónde: $\mathrm{n}=108, \mathrm{~N}=150, \alpha_{c}=95 \%, e^{2}=5 \%$

El proceso de muestreo fue tratado mediante el programa SPSS V 25 como software estadístico, en función de las variables de estudio.

\section{DISCUSIÓN DE RESULTADOS}

La tabla 1 muestra los resultados de las encuestas aplicadas para conocer lo referente a la evaluación de los registros dentales. Solo 43 de los 108 profesionales encuestados $(39,82 \%)$ almacenan los registros dentales, el 19,44\%, lo hace para el diagnóstico o tratamiento en curso y atención de seguimiento a largo plazo del paciente, solamente 9 de ellos $(8,33 \%)$ lo hacen para comparar datos dentales post y ante mortem y 7 (6,48\%) mantienen los registros como evidencia contra litigios. Mientras que, 12 de los 108 encuestados $(11,11 \%)$ indicó no 
almacenar los registros de forma personal, pero si, en la clínica odontológica para el diagnóstico y comparación dental con el $4,63 \%(n=5)$ en ambos casos. De todos los participantes $43(39,81 \%)$ indican que no mantienen los registros dentales en la clínica, ni tampoco de forma personal, sin embargo, los encuestados conocen los fines para los cuales pueden ser almacenados estos datos y pese a ello no generan su almacenaje. Tan solo $10(9,25 \%)$ de los encuestados realiza el almacenaje de forma personal, principalmente para diagnóstico y comparación dental con el $6,48 \%$ y $2,78 \%$ respectivamente.

Nuestros resultados contrastan con el estudio realizado en la India ${ }^{(4)}$ en el que se evaluó la conciencia hacia las prácticas de odontología forense, concientizando a los profesionales a mantener y compartir registros de pacientes, la mayoría de los encuestados (86\%) informó que almacenan los registros dentales de manera física o digital, siendo el archivo de fichas impresas la manera más empleada y tan solo un $29 \%$ de los encuestados registran los datos usando tecnología y archivos digitales.

Es necesario capacitar a los profesionales de nuestro país con respecto a la importancia de almacenar los registros dentales de sus pacientes para ser utilizados para la identificación forense, para de esta manera alcanzar un mayor porcentaje de lo reportado en el presente estudio, como se evidencia en una investigación realizada por Rathod y col., $2107^{(5)}$ quienes investigan un total de 100 practicantes de la rama de odontología los cuales fueron sometidos a una encuesta donde el $30 \%$ no mantiene registro alguno en sus clínicas, mientras que el $70 \%$ restante los mantienen por cualquier motivo.

Según Waleed y col., ${ }^{(6)}$ quienes realizaron una encuentra en Siria para la identificación de los datos más importantes en registros dentales, en cuanto a los datos personales el nombre y la edad del paciente son los más frecuentemente registrados con un $92 \%$ y $90 \%$ respectivamente, en cuanto al sexo un $88 \%$ y otros datos como el teléfono, la dirección o el correo electrónico el $78 \%$ registra esta información. En cuanto a los detalles clínicos el más registrado y valorado es la fecha de consulta y el diagnóstico con un $87 \%$ y $85 \%$ respectivamente, los datos que menos se registran son el examen de tejidos blandos con un $70 \%$ y el historial médico completo con un $57 \%$, este estudio muestra concordancia a la presente investigación en donde se le da una mayor importancia al registro de los datos demográficos ya que el llenado de esta sección fue satisfactorio en un $87 \%$ y a su vez la sección que menos relevancia se le da es la de los datos extraorales y los exámenes complementarios.

Se determinó ${ }^{(7)}$ que los registros dentales son mantenidos por un $86 \%$ de odontólogos y que el $36 \%$ no se sienten cómodos al ser llamados para la identificación de desastres de víctimas en masa. De acuerdo a los estudios el $79 \%$ de los dentistas mantienen registros de sus pacientes. Solo $12 \%$ de ellos mantienen registros dentales completos y apropiados para una investigación forense. En cuanto a la contribución con prácticas forenses se determinó que tan solo un $4 \%$ han contribuido a la identificación de sujetos en desastres de carácter masivo y en cuanto a las personas que han recibido un entrenamiento o preparación de carácter formal en odontología forense se encontró que el $7 \%$ es superior a la presente investigación donde solo un 3,2\% ha contribuido a investigaciones de carácter forense. 
Tabla 1.- Evaluación de registros dentales.

\begin{tabular}{|c|c|c|c|c|c|c|c|}
\hline \multirow[b]{2}{*}{$\begin{array}{l}\text { ¿Se mantienen } \\
\text { regularmente los } \\
\text { registros dentales en la } \\
\text { clínica? }\end{array}$} & \multirow[b]{2}{*}{$\begin{array}{l}\text { ¿Con que fin cree } \\
\text { usted que puede servir } \\
\text { el almacenaje de } \\
\text { registros dentales? }\end{array}$} & \multicolumn{4}{|c|}{$\begin{array}{l}\text { ¿Mantiene registros } \\
\text { dentales como insumo } \\
\text { personal? }\end{array}$} & \multirow[b]{2}{*}{ Total } & \multirow[b]{2}{*}{$\%$} \\
\hline & & Si & $\%$ & No & $\%$ & & \\
\hline \multirow{6}{*}{ Si } & $\begin{array}{l}\text { Para diagnóstico o } \\
\text { tratamiento en curso y } \\
\text { atención de seguimiento } \\
\text { a largo plazo del } \\
\text { paciente }\end{array}$ & 21 & 19,44 & 5 & 4,63 & 26 & 24,07 \\
\hline & $\begin{array}{l}\text { Comparar datos } \\
\text { dentales post y ante } \\
\text { mortem }\end{array}$ & 9 & 8,33 & 5 & 4,63 & 14 & 12,96 \\
\hline & $\begin{array}{l}\text { Administrativo o } \\
\text { cualquier otro propósito }\end{array}$ & 1 & 0,93 & 0 & 0,00 & 1 & 0,93 \\
\hline & Evidencia contra el litigio & 7 & 6,48 & 1 & 0,93 & 8 & 7,41 \\
\hline & $\begin{array}{l}\text { Objetivo de revisión del } \\
\text { caso }\end{array}$ & 2 & 1,85 & 0 & 0,00 & 2 & 1,85 \\
\hline & $\begin{array}{l}\text { Asentamientos legales } \\
\text { de casos donde se } \\
\text { necesita un certificado } \\
\text { de defunción. }\end{array}$ & 3 & 2,78 & 1 & 0,93 & 4 & 3,70 \\
\hline \multirow{5}{*}{ No } & $\begin{array}{l}\text { Para diagnóstico o } \\
\text { tratamiento en curso y } \\
\text { atención de seguimiento } \\
\text { a largo plazo del } \\
\text { paciente }\end{array}$ & 7 & 6,48 & 15 & 13,89 & 22 & 20,37 \\
\hline & $\begin{array}{l}\text { Comparar datos } \\
\text { dentales post y ante } \\
\text { mortem }\end{array}$ & 3 & 2,78 & 18 & 16,67 & 21 & 19,44 \\
\hline & $\begin{array}{l}\text { Administrativo o } \\
\text { cualquier otro propósito }\end{array}$ & 0 & 0,00 & 4 & 3,70 & 4 & 3,70 \\
\hline & Evidencia contra el litigio & 0 & 0,00 & 3 & 2,78 & 3 & 2,78 \\
\hline & $\begin{array}{l}\text { Asentamientos legales } \\
\text { de casos donde se } \\
\text { necesita un certificado } \\
\text { de defunción. }\end{array}$ & 0 & 0,00 & 3 & 2,78 & 3 & 2,78 \\
\hline Total & & 53 & 49,07 & 55 & 50,93 & 108 & 100 \\
\hline
\end{tabular}

Fuente: Instrumento de evaluación de historias clínicas procesado en SPSS v.25 
En la Tabla 2 se muestra los resultados obtenidos de las encuestas sobre la evaluación de los instrumentos de diagnóstico, observándose que en los diferentes detalles del tratamiento, los mayores valores corresponden a la categoría de los métodos de diagnóstico por radiografía sumando un total de 74 respuestas positivas lo que corresponde a un $68,51 \%$.

En el registro de tratamiento, 40 de los encuestados $(37,03 \%)$, indican mantener los resultados de las radiografías, $13,88 \%$ de ellos consideraron que su almacenaje debe hacerse por más de 5 años, y la menor cantidad (2,77\%) indicaron, que el almacenaje debería realizarse por 1 año o más.

Con respecto al plan de tratamiento 10 de los encuestados $(9,26 \%)$ indica que las radiografías deben almacenarse por más de 5 años y $6,48 \%$ refieren que el almacenaje debe hacerse por más de 10 años. La minoría (2,78\%) señaló que los registros de tratamiento con radiografía se deben guardar por más de un año.

Al analizar el descubrimiento clínico, se encuentra que solo respondieron 6 de los encuestados, de quienes la mayoría $(2,78 \%)$ manifestaron que el almacenaje debe realizarse por más de 10 años, difiriendo así de las respuestas anteriores,

Las respuestas de 23 personas $(21,29 \%)$, indican que los detalles de tratamiento de registro y plan en los rangos deben ser almacenados más de cinco y diez años, cuando se realizan los estudios de fundidos o de modelo para el diagnóstico.

Con menores proporciones se observa la importancia que le dan los encuestados 7 $(6,48 \%)$ a la fotografía clínica y $3,70 \%$ a las prueba de laboratorio.

En cuanto al tiempo de almacenamiento de los registros odontológicos nuestros resultados superan $(18,52 \%)$ a los obtenidos en el estudio en la India ${ }^{(2)}$, donde se comprueban que solo el $8 \%$ de los odontólogos, conserva los registros por más de cinco años. Estas fuentes de información son una gran herramienta para la odontología forense y el propósito de esta investigación, es que se cree conciencia del mantenimiento de los registros dentales, así como, de la entrega a las agencias gubernamentales. 
Tabla 2.- Evaluación sobre los instrumentos de diagnóstico.

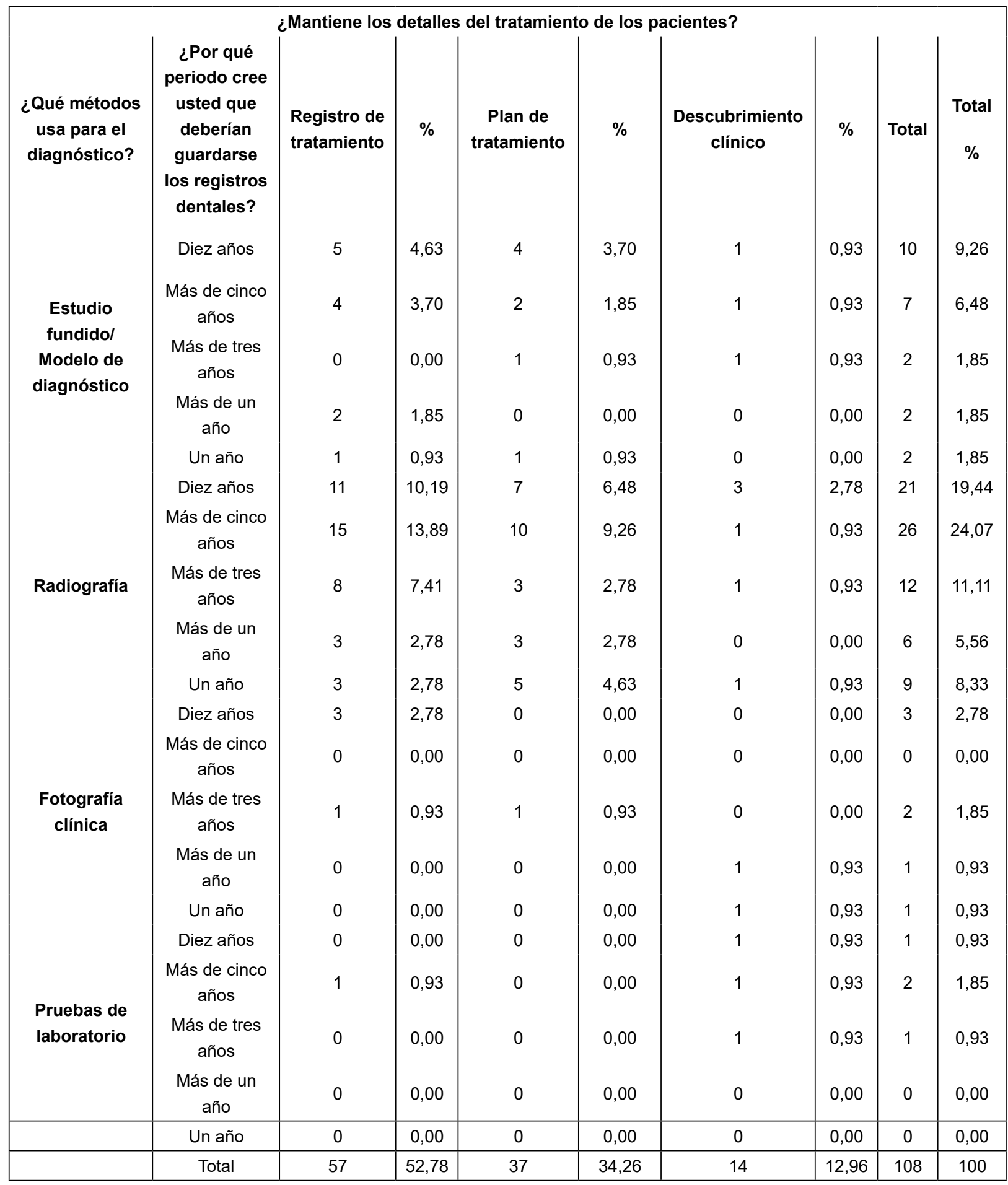

Fuente: Instrumento de evaluación de historias clínicas procesado en SPSS v.25 
La Tabla 3 muestra el análisis de conocimientos de aspectos legales. Respecto a los registros dentales requeridos para la identificación de víctimas e investigaciones forenses fueron 45 respuestas afirmativas $(41,67 \%)$ de la población de estudio relacionando a su predisposición de poner su contingente al servicio de este requerimiento aunque no se ha determinado una experiencia en la contribución de estos casos, indicando además que sí se encuentran familiarizados con tema forense y conocen el tema legal como testigo experto, solo el $20,37 \%$ de la población no conoce que puede contribuir como testigo experto, el 22,22\% (24/108) desconoce su participación como testigo experto y no tiene familiaridad con el tema forense, y tampoco ha contribuido en la identificación de víctimas. Solo 5 personas, lo que corresponde al $4,63 \%$, indican que no compartiría registros dentales en un elemento de requerimiento sobre una investigación forense. Además, un pequeño porcentaje $(2,78 \%)$ indicó la predisposición de compartir sus registros, que ha contribuido con la identificación de víctimas, la familiaridad con el tema forense y conoce del tema legal.

Por lo tanto nuestros resultados, difieren a los obtenidos en un estudio ${ }^{(4)}$, en donde encontraron que los participantes $15 \%$, manifestaron que mantendrán registros en el futuro y un alto porcentaje $64 \%$ no se sienten cómodos en compartir estos datos de registros almacenados. Mientras que el $51,6 \%$ de los profesionales en la Clínica Odontológica de la Unach, mantienen registros dentales y la gran mayoría 93,52\% está dispuesto a colaborar compartiendo los datos de sus pacientes en caso de ser requeridos.

Tabla 3.- Análisis de conocimientos de aspectos legales.

\begin{tabular}{|c|c|c|c|c|c|c|c|c|}
\hline \multirow[b]{2}{*}{$\begin{array}{l}\text { Si fuera requerido, } \\
\text { ¿compartiría los } \\
\text { registros dentales } \\
\text { de su paciente } \\
\text { con una agencia } \\
\text { gubernamental para } \\
\text { ayudar a identificar } \\
\text { a las víctimas de } \\
\text { desastres masivos? }\end{array}$} & \multirow[b]{2}{*}{$\begin{array}{l}\text { ¿Ha contribuido } \\
\text { alguna vez a la } \\
\text { identificación } \\
\text { de víctimas } \\
\text { en desastres } \\
\text { masivos? }\end{array}$} & \multirow[b]{2}{*}{$\begin{array}{l}\text { ¿Está } \\
\text { familiarizado } \\
\text { con el } \\
\text { tema de la } \\
\text { medicina } \\
\text { forense y } \\
\text { su relación } \\
\text { con la } \\
\text { odontología? }\end{array}$} & \multicolumn{6}{|c|}{$\begin{array}{l}\text { ¿Sabe que puede declarar como testigo experto en el } \\
\text { tribunal para presentar pruebas forenses? }\end{array}$} \\
\hline & & & Si & $\begin{array}{c}\text { Porcentaje } \\
\%\end{array}$ & No & $\begin{array}{c}\text { Porcentaje } \\
\quad \%\end{array}$ & Total & $\begin{array}{c}\text { Total } \\
\text { Porcentaje } \\
\text { \% }\end{array}$ \\
\hline \multirow{4}{*}{$\mathrm{Si}$} & \multirow{2}{*}{$\mathrm{Si}$} & $\mathrm{Si}$ & 3 & 2,78 & 0 & 0,00 & 3 & 2,78 \\
\hline & & No & 0 & 0,00 & 1 & 0,93 & 1 & 0,93 \\
\hline & \multirow{2}{*}{ No } & $\mathrm{Si}$ & 45 & 41,67 & 22 & 20,37 & 67 & 62,04 \\
\hline & & No & 6 & 5,56 & 24 & 22,22 & 30 & 27,78 \\
\hline \multirow{3}{*}{ No } & \multirow{2}{*}{ No } & $\mathrm{Si}$ & 1 & 0,93 & 1 & 0,93 & 2 & 1,85 \\
\hline & & No & 2 & 1,85 & 3 & 2,78 & 5 & 4,63 \\
\hline & & Total & 57 & 52,78 & 51 & 47,22 & 108 & 100 \\
\hline
\end{tabular}

Fuente: Instrumento de evaluación de historias clínicas procesado en SPSS v.25 
En la Tabla 4 se registra el conocimiento de odontología forense, la evaluación de conocimiento sobre el identificación forense y estimación de la edad se pudo observar que la frecuencia más alta corresponde a 50 personas lo que equivale a un $46,29 \%$ que indicaron haber sido formados en el aspectos de odontología forense y cuyos ítems fueron contestados de manera correcta. Se puede apreciar que personas que dicen no haber sido formadas en forense mostraron certeza en una de las preguntas con un $10,19 \%$ y $13,89 \%$, El $24 \%$ de los encuestados mostraron una valoración de desacierto en el conocimiento de los ítems planteados, pese que el $11 \%$ señala que tuvo formación en el tema, finalmente el $23 \%$ de los encuestados contestaron correctamente uno de los ítems sin tener ninguna formación.

En estudio reciente ${ }^{(5)}$ se describe que el $50 \%$ de los encuestados manifestaron que no saben cómo identificar abuso infantil, mientras que, el resto declaró que se puede realizar por medio de lesiones, marcas, comportamiento anormal, vestimenta o la manera de alimentación. Solo el $5 \%$ manifestó que han sido parte de un equipo forense en sus respectivas ciudades, alrededor del $75 \%$ no son conscientes de que pueden testificar como expertos en una corte, el $15 \%$ de los encuestados han tenido entrenamiento en colección, evaluación y presentimiento de evidencias. Por último, el $75 \%$ de los participantes no confían en involucrarse con casos de ciencias forense, por lo tanto se puede encontrar similitudes en este estudio en donde existe un desconocimiento de que un odontólogo puede declarar como testigo experto en un tribunal ${ }^{(5)}$.

La mayoría de los profesionales de odontología reconoce que las técnicas de reconocimiento de ADN son las más sensibles y específicas como ha sido comprobado por Waleed y col. ${ }^{(6)}$ que indican que el $65 \%$ de sus encuestados manifestaron conocer la sensibilidad y eficacia de una muestra de ADN o de huellas dactilares, para determinar la identificación de una persona.

Tabla 4. Conocimiento de odontología forense.

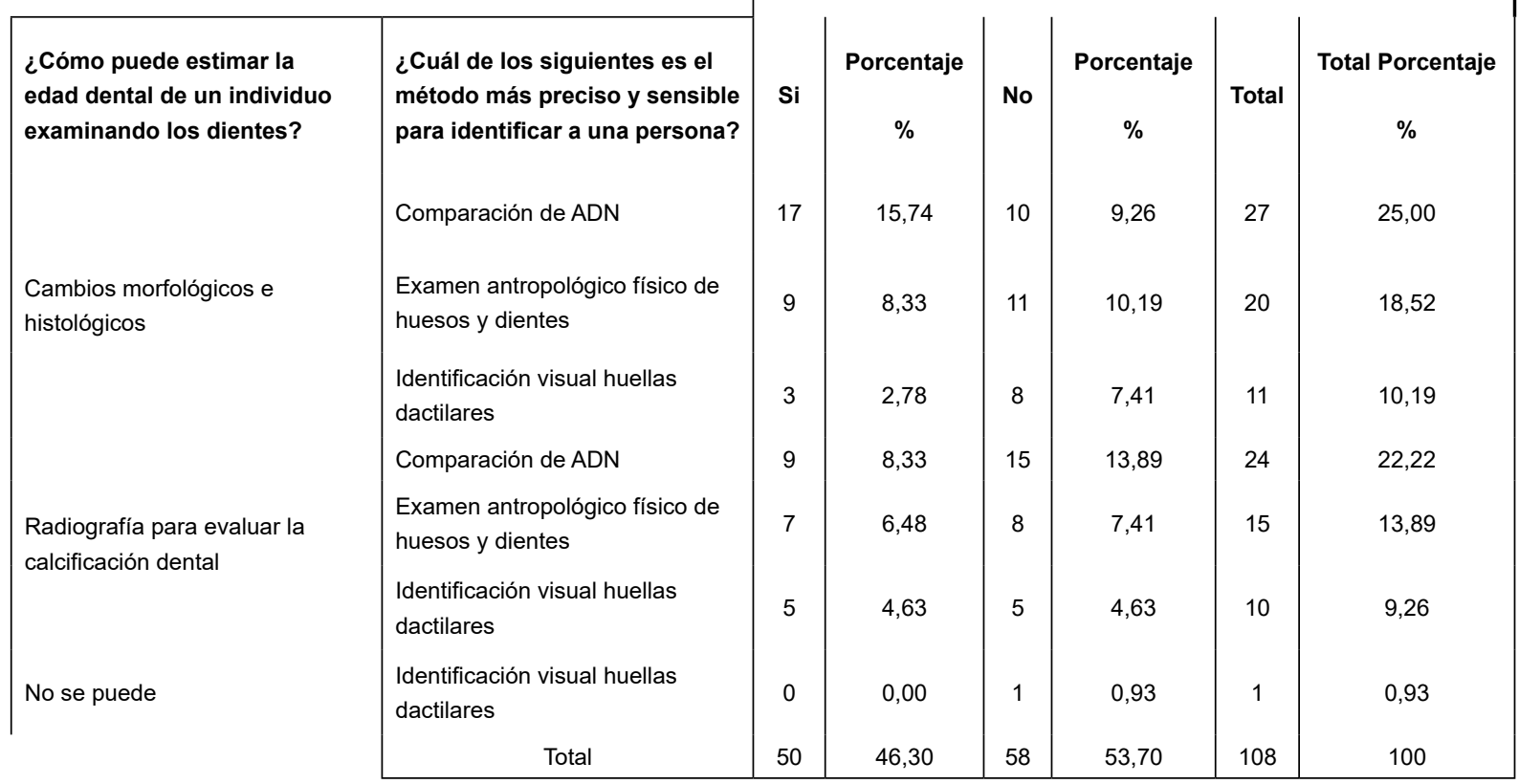

Fuente: Instrumento de evaluación de historias clínicas procesado en SPSS v.25 
La Tabla 5 muestra el conocimiento sobre marcas de mordida, los resultados indican que el $66,67 \%$ de ellos conocen la relación entre la medicina forense y la odontología. Se aprecia que 13 de ellos (12,03\%) determinaron que las marcas se generan por dientes humanos con lesiones de laceración, así como el 11,11\% determinado por moretones, el 7,41\% menciona que la importancia de las marcas de mordidas, está dada por la orientación y ubicación de la marca en referencia a la laceración como lesión. Solo 36 personas $(33,33 \%)$ indicaron que no se encuentran familiarizados con los temas de medicina forense. Mientras que la mayoría de este grupo, 7 encuestados (6,48\%) refieren la importancia de las marcas de los dientes humanos y animales cuya lesión conlleva a laceración. El resto de ítems fueron contestado en sus diferentes opciones siendo la tendencia del grupo una valoración del hasta el 3,70\% de diferentes criterios tanto para la lesión como para la importancia de las marcas; para este análisis se ha considerado las valoraciones más altas en la tendencia de la población tomando en cuenta que cada uno de los ítems tiene su priorización en el criterio de cada encuestado.

Los profesionales de odontología tienen mucho más conocimiento de la información que pueden obtenerse a partir de las marcas de mordidas y el $27 \%$ de los encuestados han tenido capacitación en recopilar evaluar y presentar evidencias a diferencia del $15 \%$ registrado en el estudio de Rathod y col. ${ }^{(5)}$

La Tabla 6 muestra el conocimiento para reconocimiento de técnicas, se puede observar que la mayoría de las personas no conocen ninguna de las mismas, el método de palatoscopía / rugoscopía palatina usado para determinación del sexo al igual que la reacción en cadena de la polimerasa no es conocido en el $83,3 \%$ de la población de estudio. Finalmente se les consultó si han conocido la resolución de alguna investigación mediante métodos de odontología forense siendo el $76,9 \%$ de las respuestas negativas.

Tabla 5.- Conocimiento sobre marcas de mordida

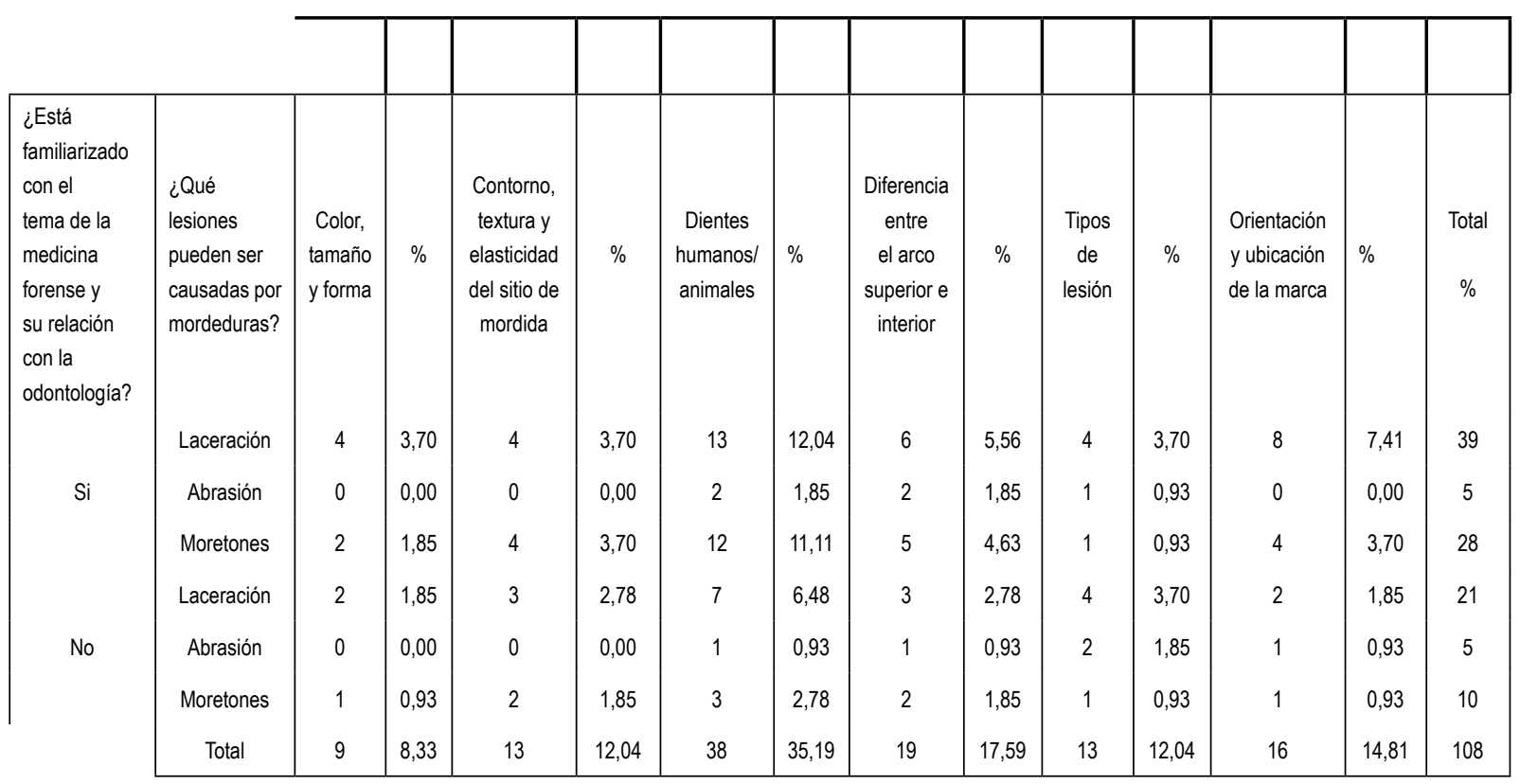

Fuente: Instrumento de evaluación de historias clínicas procesado en SPSS v.25 
Tabla 6.- Conocimiento para reconocimiento de técnicas.

\begin{tabular}{|c|c|c|}
\hline $\begin{array}{c}\text { ¿Conoce el método de palatoscopía / rugoscopía } \\
\text { palatina utilizado para determinar el sexo en } \\
\text { individuos? }\end{array}$ & Frecuencia & Porcentaje (\%) \\
\hline Si & 18 & 16,7 \\
\hline No & 90 & 83,3 \\
\hline $\begin{array}{c}\text { ¿Conoce los métodos de PCR (reacción en cadena } \\
\text { de la polimerasa) utilizados para identificar la } \\
\text { relación genética entre los individuos? }\end{array}$ & Frecuencia & Porcentaje (\%) \\
\hline Si & 18 & 16,7 \\
\hline No & 90 & 83,3 \\
\hline $\begin{array}{c}\text { ¿Tiene conocimiento de alguna investigación } \\
\text { en el país que haya sido resuelta por métodos } \\
\text { odontología forense? }\end{array}$ & Frecuencia & Porcentaje (\%) \\
\hline Si & 25 & 23,1 \\
\hline No & 83 & 76,9 \\
\hline
\end{tabular}

Fuente: Instrumento de evaluación de historias clínicas procesado en SPSS v.25

En la Tabla 7 se muestra la capacitación en odontología forense, evidenciando que la mayoría de las personas no están capacitados alcanzando un $70,37 \%$ de los encuestados, sin embargo, el $94,44 \%$ de ellos mostraron gran interés en capacitarse en aspectos de odontología forense dentro del país, mientras que, el $24,07 \%$ indica que ha tenido una capacitación y está dispuesto a continuar estudios para su formación forense.

Tabla 7.- Capacitación en odontología forense.

\begin{tabular}{|c|c|c|c|c|c|c|}
\hline \multirow[b]{2}{*}{$\begin{array}{c}\text { ¿Ha tenido alguna capacitación } \\
\text { formal para recopilar, evaluar y } \\
\text { presentar evidencia dental? }\end{array}$} & \multicolumn{6}{|c|}{$\begin{array}{l}\text { ¿Le gustaría hacer un curso de capacitación en } \\
\text { odontología forense si tiene posibilidades dentro del país? }\end{array}$} \\
\hline & Si & $\begin{array}{c}\text { Porcentaje } \\
\%\end{array}$ & No & $\begin{array}{c}\text { Porcentaje } \\
\%\end{array}$ & Total & $\begin{array}{c}\text { Total } \\
\text { Porcentaje } \\
\%\end{array}$ \\
\hline Si & 26 & 24,07 & 4 & 3,70 & 30 & 27,78 \\
\hline No & 76 & 70,37 & 2 & 1,85 & 78 & 72,22 \\
\hline Total & 102 & 94,44 & 6 & 5,56 & 108 & 100,00 \\
\hline
\end{tabular}

Fuente: Instrumento de evaluación de historias clínicas procesado en SPSS v.25

El Gráfico 1 se muestra la evaluación de datos demográficos, al analizar el componente de historia clínica evaluando los ítems que mejor contribuyan a un análisis forense, mediante el análisis individual de los historiales clínicos, se pudo observar que el nivel de información de estos documentos fue satisfactorio para la mayor parte de los encuestados $(81,5 \%)$. 
Gráfico 1.- Evaluación de datos demográficos

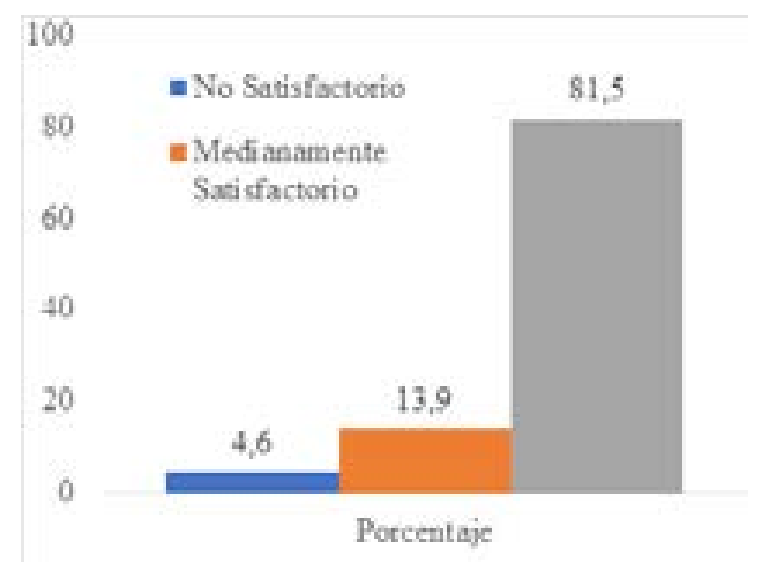

Fuente: Instrumento de evaluación de historias clínicas procesado en SPSS v.25

El Grafico 2, muestra la satisfacción de los encuestados de acuerdo a las preguntas de los datos intraorales, respecto a la evaluación de los datos intraorales se pudo apreciar que para la mayoría $60,2 \%$ resultaron medianamente satisfactorias.

\section{Gráfico 2.- Datos intraorales}

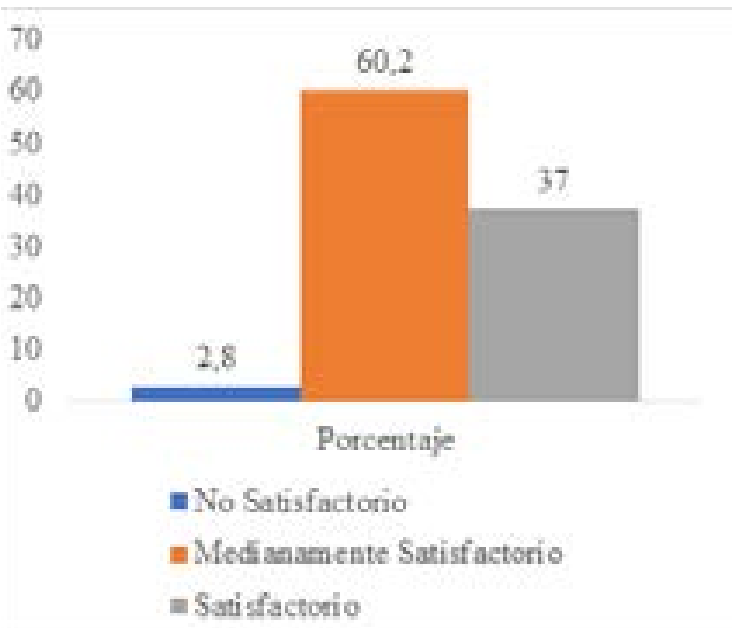

Fuente: Instrumento de evaluación de historias clínicas procesado en SPSS v.25

El Grafico 3, muestra el nivel de satisfacción de los encuestados de acuerdo a las preguntas de los datos extraorales, se puede observar que el valor no satisfactorio fue el de mayor presencia en la población con el $51,9 \%$ de historiales clínico cuya información no cumple con lo necesario para una investigación de tipo forense.

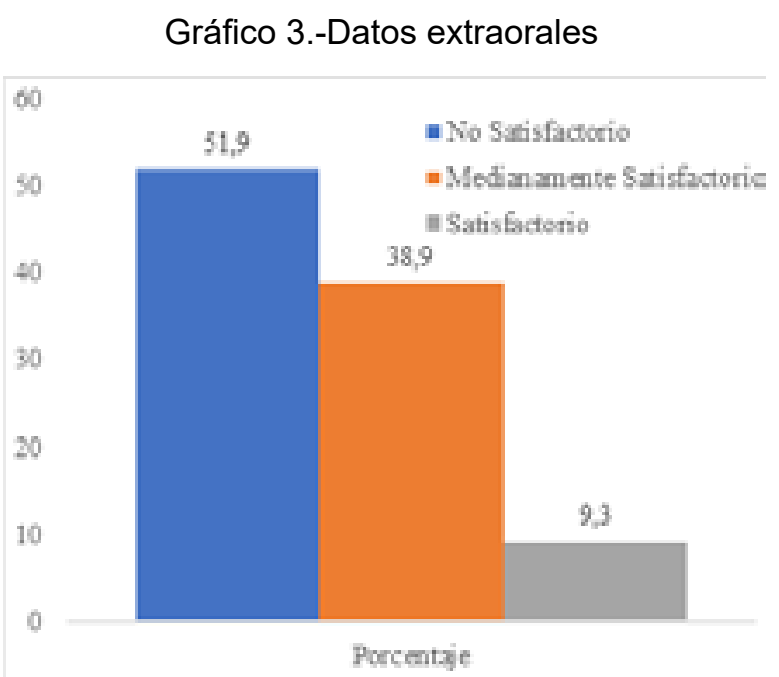

Fuente: Instrumento de evaluación de historias clínicas procesado en SPSS v.25

El grafico 4 muestra el grado de satisfacción respecto a la evaluación del registro de exámenes complementarios, el $63 \%$ indica que los valores no fueron satisfactorios para fines forenses.

Gráfico 4 Registro de exámenes complementarios

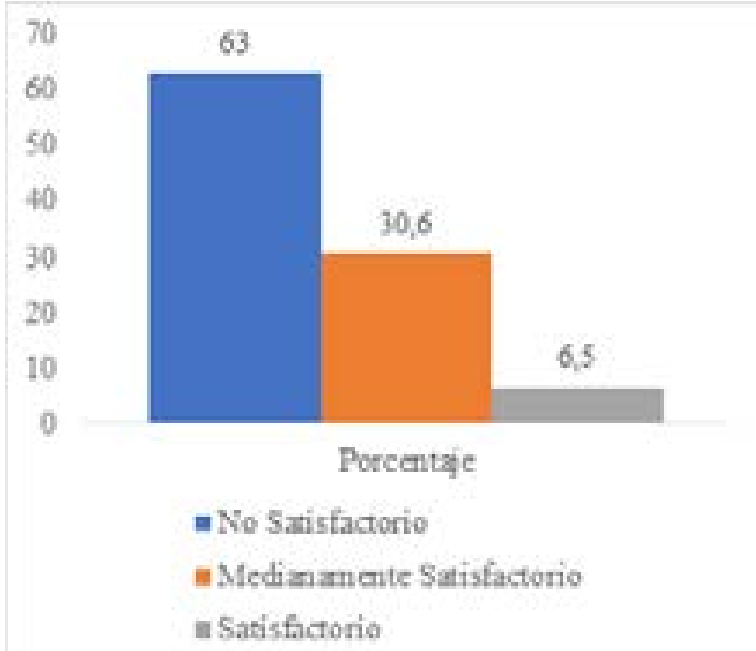

Fuente: Instrumento de evaluación de historias clínicas procesado en SPSS v. 25

El Gráfico 5 muestra el registro de avances de la historia clínica, alcanzó un nivel de satisfacción del $76,9 \%$ en los historiales. 
Gráfico 5.- Registro de avances

de la historia clínica

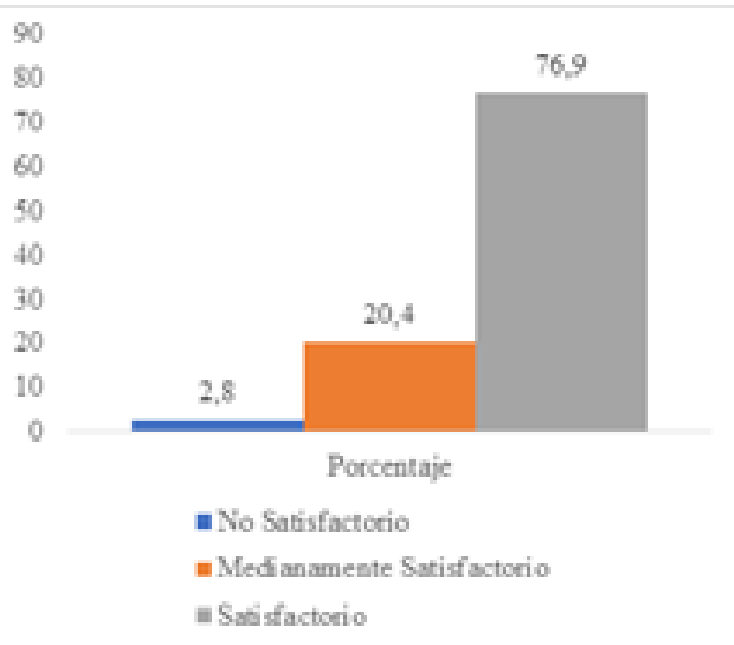

Fuente: Instrumento de evaluación de historias clínicas procesado en SPSS v. 25

En base al análisis realizado en la presente investigaciónsediseñólaficha deimplementación de datos del paciente para la investigación forense mediante su adaptación en la historia clínica (Tabla 8 ).Esta ficha de implementación cumple con un rol fundamental, debido a que bajo los criterios de observación y correlación pudimos identificar que datos y características son requeridos, además de los ya encontrados y usados en el formulario 033 (Historia clínica única de odontología) del Ministerio de Salud
Pública acerca de las características físicas del paciente y morfológicas de la cavidad bucal específicamente, debido a que cada persona cuenta con variaciones y alteraciones que pueden ser características de su desarrollo así como por medio de los tratamientos dentales realizados a lo largo de su vida y que deben ser representados específicamente con simbología única en base al tratamiento realizado. De igual manera se debe implementar una cadena de custodia en donde se registre el responsable del almacenaje de la ficha de registro así como observaciones de características únicas encontradas en el paciente.

Por otra parte se realizó la validación de esta ficha de registro ( anexo 3 ) en base al trabajo conjunto del Departamento de Ciencias Forenses y de conocimientos odontológicos basados en que datos pueden ser beneficiosos para realizar investigaciones de carácter médico-legales y nos puedan ayudar a solventar la complejidad de ciertos casos mediante las características y alteraciones morfológicas encontradas en la cavidad bucal ya que se comprueba que no existe dos individuos con configuraciones esquelético-dentales y morfológicas idénticas. 
Tabla 8.- Ficha de implementación de datos del paciente para la investigación forense mediante su adaptación en la historia clínica.

\begin{tabular}{|c|c|}
\hline Datos del paciente HC & Datos faltantes en la HC \\
\hline $\begin{array}{l}\text { Nombre } \\
\text { Fecha de nacimiento } \\
\text { Nacionalidad } \\
\text { Dirección de domicilio } \\
\text { Ocupación }\end{array}$ & $\begin{array}{l}\text { Cédula de Identidad } \\
\text { Pasaporte }\end{array}$ \\
\hline Características físicas generales HC & Características físicas complementarias \\
\hline $\begin{array}{l}\text { Género } \\
\text { Edad } \\
\end{array}$ & $\begin{array}{l}\text { Estatura del paciente } \\
\text { Registro de la ancestría o afinidad racial } \\
\end{array}$ \\
\hline Datos intraorales HC & Datos intraorales complementarios \\
\hline $\begin{array}{l}\text { No existe una representación gráfica, y de } \\
\text { simbología específica para registrar los diferentes } \\
\text { hallazgos dentarios con diferenciación de sus } \\
\text { materiales y características. }\end{array}$ & $\begin{array}{l}\text { Dibujo o croquis de las dos arcadas dentarias } \\
\text { con una vista de todas sus caras y bordes, con } \\
\text { la corona y raíz correspondientes. } \\
\text { Simbología: existe una gran variedad de } \\
\text { tratamientos y diferentes materiales dentales los } \\
\text { cuales se deben describir con mayor detalle en } \\
\text { su caracterización y simbología. }\end{array}$ \\
\hline Registro de exámenes complementarios HC & Datos de registro complementario. \\
\hline $\begin{array}{l}\text { Pruebas de laboratorio } \\
\text { Radiografías } \\
\text { Registro fotográfico } \\
\text { Modelos de estudio }\end{array}$ & Responsable del almacenaje \\
\hline Interpretación narrativa HC & Interpretación narrativa complementaria \\
\hline No existe & $\begin{array}{l}\text { Renglones de detalle breve de hallazgos por } \\
\text { cuadrante }\end{array}$ \\
\hline Observaciones HC & Observaciones \\
\hline $\begin{array}{l}\text { Solo se detallan aspectos referentes al } \\
\text { diagnóstico }\end{array}$ & $\begin{array}{l}\text { Reglón adicional en la cual se detallará } \\
\text { anomalías específicas y características únicas } \\
\text { del paciente }\end{array}$ \\
\hline
\end{tabular}




\section{CONCLUSIONES}

- El grado de conocimiento sobre odontología forense se midió a partir de una encuesta de 20 pregunta, en la cual se pudo evaluar los registros dentales y su utilidad. Se destaca que solo el $8 \%$ de los encuestados manifiestan que al mantener los registros pueden ser útiles para la comparación de datos ante y post mortem, en cuanto a los aspectos legales el 95\% afirma que compartiría los registros de los pacientes en el caso de ser requeridos para investigación forense, y destaca que el $94 \%$ de los encuestados muestran un alto interés en capacitarse en esta especialidad.

- Se identificaron las fortalezas y debilidades en el llenado de las historias clínicas actualmente usadas. En el análisis de datos demográficos se demostró que la recolección de datos del paciente podían ser almacenados satisfactoriamente por el $81,5 \%$, siendo de utilidad en nuestra investigación. Y en cuanto a la recopilación de datos intraorales el $60,2 \%$ fue medianamente satisfactorio por considerarse la sección más importante, se debe corregir la manera como está estructurada.

- Se diseñó una propuesta de ficha odontológica que en función de la validación realizada por los expertos, permite manejar la información con fines de carácter forense, para que en las investigaciones que sean requeridas puedan tener la facilidad $y$ acceso a esta información. A su vez, con la difusión de la presente investigación y el planteamiento de la ficha de registro determinado en el anexo 2, se busca contribuir para que el proceso de recolección de datos en la historia clínica, sea un instrumento adicional que permita crear una cultura en la recopilación de información válida para investigaciones forenses.

\section{REFERENCIAS BIBLIOGRÁFICAS}

1. Lizbeth de las Mercedes Rodríguez La ficha de identificación estomatológica para internos: un asunto aún pendiente en México. Rev. Crim. vol.54 no.2 Bogotá July/ Dec. 2012

2. Maestud M, Melo A. REVISIÓN DE LA LITERATURA DETERMINATION OF THE REAL AGE THROUGH RECORDS OBTAINED IN THE DENTAL CLINIC. A LITERATURE REVIEW.

3. Hinchliffe J. Forensic odontology, part 1. Dental identification. Br Dent J [Internet]. 2011 Mar 12 [cited 2018 Dec 4];210(5):21924. Available from: http://www.nature.com/ articles/sj.bdj.2011.146

4. Sengupta S, Sharma V, Vij H, Vij R, Prabhat K, Gupta V. Forensic odontology as a victim identification tool in mass disasters: A feasibility study in the Indian scenario. J Forensic Dent Sci. 2014.

5. Rathod V, Desai V, Pundir S, Dixit S, Chandraker R. Role of forensic dentistry for dental practitioners: A comprehensive study. J Forensic Dent Sci. 2017.

6. Waleed P, Baba F, Alsulami S, Tarakji B. Importance of dental records in forensic dental identification. Acta Inform Medica. 2015.

7. Gambhir R, Singh G, Talwar P, Gambhir $J$, Munjal V. Knowledge and awareness of forensic odontology among dentists in India: A systematic review. J Forensic Dent Sci. 2016;8(1):2.

8. Balla SB. Forensic Dental Identification: Practice in Indian Context Compared to Western Countries. J Forensic Sci Med 2016;2:44-7.

9. Krishan K, Kanchan T, Garg AK. Dental 
Evidence in Forensic Identification - An Overview, Methodology and Present Status. Open Dent J [Internet]. 2015;9(1):250-6. Available from: http://benthamopen.com/ ABSTRACT/TODENTJ-9-250

10. Fonseca GM, Cantín M, Lucena J. Odontología Forense III: Rugas Palatinas y Huellas Labiales en Identificación Forense. Int J Odontostomat. 2014;8(1):29-40.

11. Somaraj V, Ravishankar $P$, Agnes A, Vaithiswari A, Magdalene B, Bell MGB. Forensic Odontology - A Science within a Science. 2018;1-4.

12. Caldas IM, Magalhães $T$, Afonso $A$. Establishing identity using cheiloscopy and palatoscopy. Forensic Sci Int. 2007;165(1):1-9.

13. Hinchliffe J. Forensic odontology, part 2. Major disasters. Br Dent J [Internet]. 2011 Mar 26 [cited 2018 Dec 4];210(6):269-74. Available from: http://www.nature.com/ articles/sj.bdj.2011.199

14. Schuller-Götzburg P, Suchanek J. Forensic odontologists successfully identify tsunami victims in Phuket, Thailand. Forensic Sci Int. 2007;171(2-3):204-7.

15. Zakirulla M, Allahbaksh M. Modern Tools in Forensic Dentistry. Int J Contemp Dent. 2011;2(3):28-33.

16. Raimann PE, Picanc JB, Silva DSBS, Jackson F, Paludo O, Alho CS. Procedures to recover DNA from pre-molar and molar teeth of decomposed cadavers with different post-mortem intervals. 2012;7:3-10.

17. Zorba E, Spiliopoulou C, Moraitis K. Evaluation of the accuracy of different molar teeth measurements in assessing sex. Forensic Sci Med Pathol. 2013;9(1):13-23.
Macedo Í De. Archives of Oral Biology Reliability of palatal rugoscopy for sexual dimorphism in forensic dentistry: A systematic literature review and metaanalysis. Arch Oral Biol [Internet]. 2019;97(September 2018):25-34. Available from: https://doi.org/10.1016/j. archoralbio.2018.10.009

19. Porto IM, Laure HJ, Tykot RH, de Sousa FB, Rosa JC, Gerlach RF. Recovery and identification of mature enamel proteins in ancient teeth. Eur $\mathrm{J}$ Oral Sci. 2011;119(SUPPL.1):83-7.

20. Silva RF, Franco A, Picoli FF, Rodrigues LG, Resende RG. Dental human identifi cation using bitewing radiographs: A case report. 2016;3(3):31-3.

21. Krishan K, Kanchan T, Garg AK. Dental Evidence in Forensic Identification - An Overview, Methodology and Present Status. Open Dent J [Internet]. 2015 [cited 2018 Dec 4];9:250-6. Available from: http://www. ncbi.nlm.nih.gov/pubmed/26312096

22. Tapia RP, Lovón WE. Evaluación odontológica forense de huellas de mordida: reporte de un caso. 2015;1(3):36-9.

23. Chokhani S. DENTAL RECORD MAINTENANCE - ITS IMPORTANCE IN FORENSIC ODONTOLOGY AND MEDICOLEGAL. 2017;2(6):126-31.

24. Astekar M. Maintaining dental records : Are we ready for forensic needs ? 2011;3(2):38. 
Anexo 1. Simbología utilizada en las historia clínicas

\begin{tabular}{lll}
\hline Simbología & Color & Referencia \\
\hline Gráficos o símbolos & Para distinguir Observaciones correspondientes \\
usados & $\begin{array}{l}\text { los tratamientos y } \\
\text { materiales }\end{array}$ \\
\hline
\end{tabular}

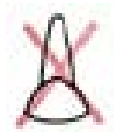

Rojo

Piezas ausentes ante mortem

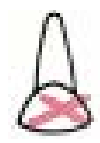

Rojo

Restos radiculares



Rojo

Ausencia clínica de la cavidad, sin poder establecer si la pieza se encuentra incluida o perdida y sin el auxilio de los rayos $X$

Rojo

Fractura coronaria, delinear el trazo de la fractura

Rojo

Fractura de maxilar delinear el trazo de la fractura

Rojo

Fractura de mandíbula delinear el trazo de la fractura

Rojo

Cavidades cariosas marcar áreas afectadas

Preparación de cavidad con obturación temporal marcar áreas afectadas

Negro

Restauración de amalgama o incrustaciones metálicas 


\begin{tabular}{lll}
\hline Simbología & Color & Referencia \\
\hline Gráficos o símbolos & Para distinguir & Observaciones correspondientes \\
usados & $\begin{array}{l}\text { los tratamientos y } \\
\text { materiales }\end{array}$ \\
\hline
\end{tabular}

Azul

Negro

Amarillo

Azul

Amarillo

Negro

Anaranjado

0

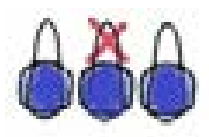

㩆

Rojo
Restauración de resina o incrustaciones estéticas

Coronas $3 / 4$ de metal plateado

Coronas $3 / 4$ de oro

Coronas totales de materiales estéticos

Coronas totales de oro

Coronas totales de metal

Coronas provisionales

\section{Según corresponda Prótesis fija}

Prótesis parcial removible, anotar características y color correspondiente 


\begin{tabular}{lll}
\hline Simbología & Color & Referencia \\
\hline Gráficos o símbolos & Para distinguir & Observaciones correspondientes \\
usados & $\begin{array}{l}\text { los tratamientos y } \\
\text { materiales }\end{array}$ \\
\hline
\end{tabular}

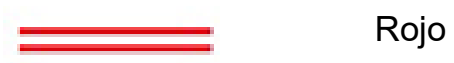

Negro

Negro

Rojo

Negro
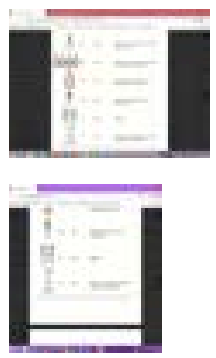

Negro

Negro
Prótesis total, anotar características y color correspondiente

Tratamiento de endodoncia, marcando el conducto tratado

Tratamiento de ortodoncia

Piezas incluidas

Desgaste dentario, delinear la zona correspondiente

Diastemas

Mal posiciones, indicando hacia donde se encuentran con la flecha correspondiente

Elaborado por: Marcelo Mancheno

Adaptado de: Correa, Alberto Isaac; Estomatología forense 
Anexo 2 Ficha de registro odontológico con fines forenses

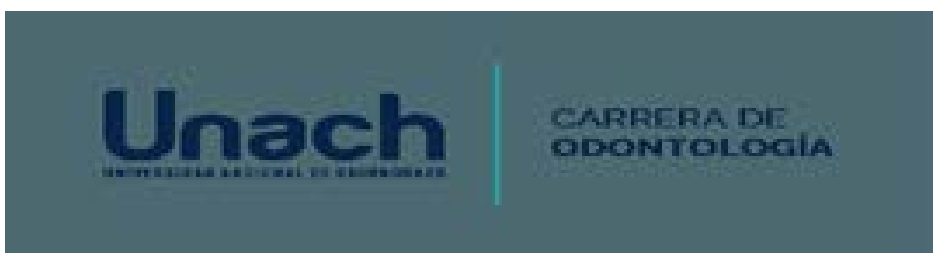

Ficha de registro odontológico con fines forenses

1. Datos generales

Nombre:

Fecha de nacimiento:

Cl/PAS:

Dirección:

2. Características físicas generales

Sexo:

Estatura
Nacionalidad:

Ocupación:

Edad:

Ancestría:

3.
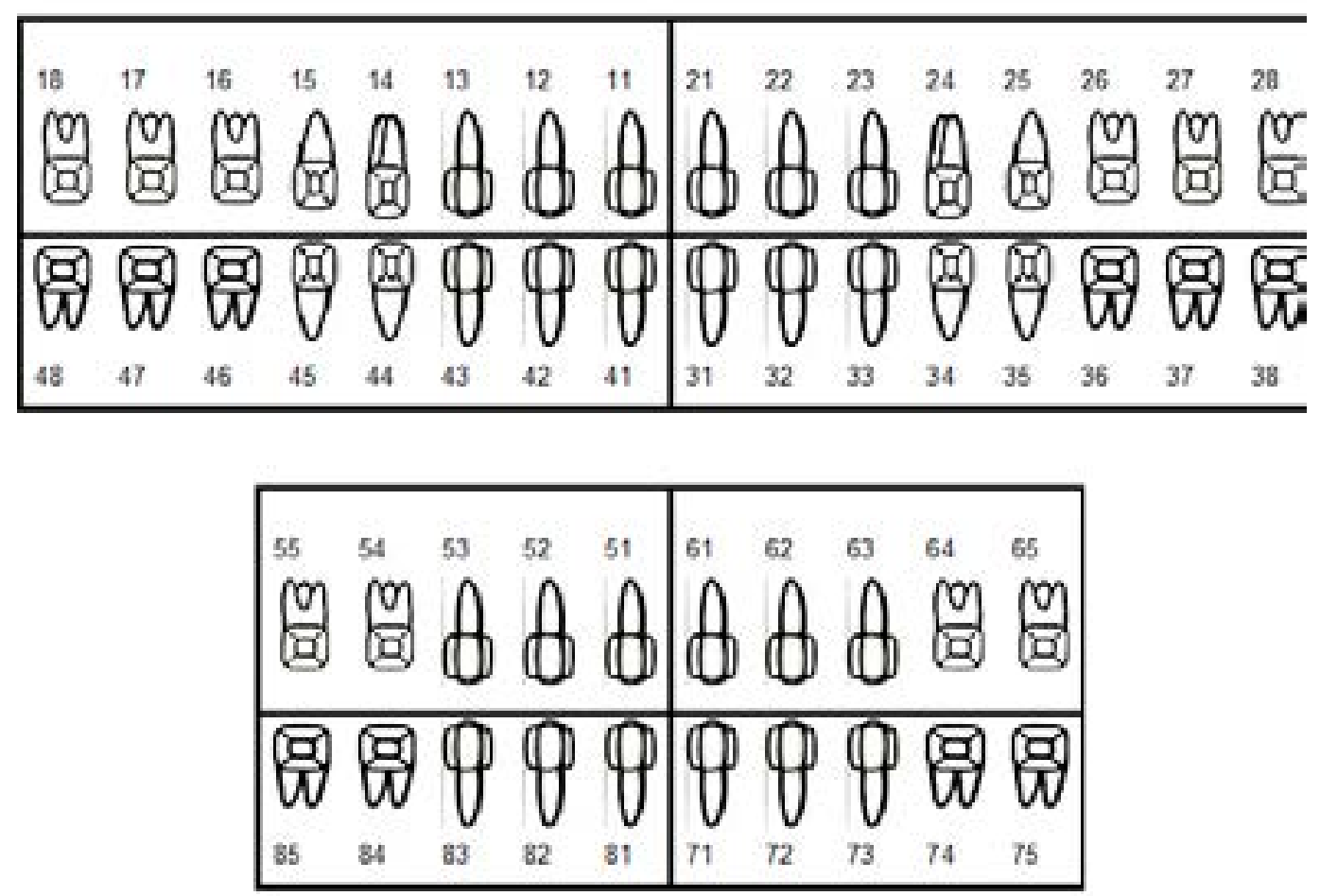
4. Exámenes complementarios

Modelos de estudio: SI__ NO

Tipo:

Fotografías: SI

NO_Responsable:

RX: SI NO Tipo:

Responsable:

5. Interpretación

Cuadrante superior derecho 11.

12.

13.

14.

15.

16.

17.

18.

Cuadrante inferior derecho

41.

42.

43.

44.

45.

46.

47.

48.

6. Observaciones
Cuadrante superior izquierdo

21.

22.

23.

24.

25.

26.

27.

28.

Cuadrante inferior izquierdo

31.

32.

33.

34.

35.

36.

37.

38. 
Anexo 3. Ficha de validación por expertos

Ficha de validación por expertos

Nombre: Anoveler

Apellido: Gatar 20

Experiencia laboral:
Grado académico: Médica Forense Fecha de revisión: 22 Mayo 2019 6 a ños

\section{Aspectos de validación}

\begin{tabular}{|c|c|c|c|c|}
\hline Nro. & Aspectos para considerar & Cumple & No Cumple & Observación \\
\hline 1 & $\begin{array}{l}\text { La ficha de registro cuenta con } \\
\text { suficiente información necesaria para } \\
\text { una posible investigación de carácter } \\
\text { forense. }\end{array}$ & & & \\
\hline 2 & $\begin{array}{l}\text { Existe claridad acerca de la utilidad de } \\
\text { la ficha de registro odontológico }\end{array}$ & & & \\
\hline 3 & $\begin{array}{l}\text { La ficha de registro tiene coherencia } \\
\text { con respecto al objetivo de su uso }\end{array}$ & & & \\
\hline 4 & $\begin{array}{l}\text { Cree usted que la ficha de registro } \\
\text { tenga la importancia y relevancia } \\
\text { necesaria. }\end{array}$ & & & \\
\hline 5 & $\begin{array}{l}\text { La ficha de registro tiene facilidad y } \\
\text { toma poco tiempo en el llenado de la } \\
\text { misma }\end{array}$ & & & \\
\hline 6 & $\begin{array}{l}\text { Cree usted que estos datos permitirán } \\
\text { tener ayuda en casos de investigación }\end{array}$ & & & \\
\hline
\end{tabular}

Observaciones generales:

Validado por:

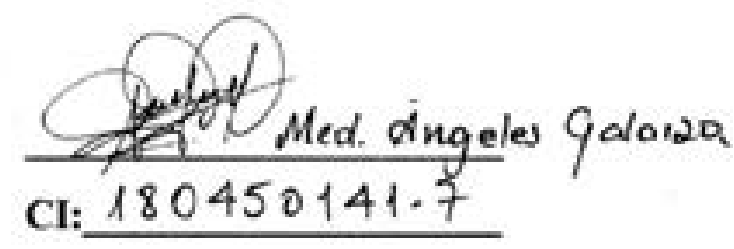


Ficha de validación por expertos

Nombre: Jéssico Rola Grado académico:

Tercer Nivel

Apellido: Machado Muño2 Fecha de revisión: $22 / 05 / 20 / 9$ Experiencia laboral:

4 años

\section{Aspectos de validación}

\begin{tabular}{|c|c|c|c|c|}
\hline Nro. & Aspectos para considerar & Cumple & No Cumple & Observación \\
\hline 1 & $\begin{array}{l}\text { La ficha de registro cuenta con } \\
\text { suficiente información necesaria para } \\
\text { una posible investigación de carácter } \\
\text { forense. }\end{array}$ & & & \\
\hline 2 & $\begin{array}{l}\text { Existe claridad acerca de la utilidad de } \\
\text { la ficha de registro odontológico }\end{array}$ & & & \\
\hline 3 & $\begin{array}{l}\text { La ficha de registro tiene coherencia } \\
\text { con respecto al objetivo de su uso }\end{array}$ & & & \\
\hline 4 & $\begin{array}{l}\text { Cree usted que la ficha de registro } \\
\text { tenga la importancia y relevancia } \\
\text { necesaria. }\end{array}$ & & & \\
\hline 5 & $\begin{array}{l}\text { La ficha de registro tiene facilidad y } \\
\text { toma poco tiempo en el llenado de la } \\
\text { misma }\end{array}$ & $\gamma$ & & \\
\hline 6 & $\begin{array}{l}\text { Cree usted que estos datos permitirán } \\
\text { tener ayuda en casos de investigación }\end{array}$ & $\gamma$ & & \\
\hline
\end{tabular}

Observaciones generales:

Validado por:

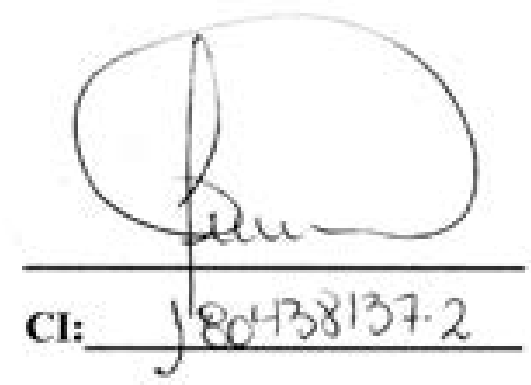


Ficha de validación por expertos

Nombre: Iorge Marcelo Grado académico:

Apellido: Quintom Y Ýánez Fecha de revisión:

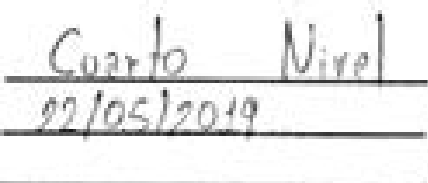

Experiencia laboral:

$6 \mathrm{\partial n \overline {O }}$

Aspectos de validación

\begin{tabular}{|c|c|c|c|c|}
\hline Nro. & Aspectos para considerar & Cumple & No Cumple & Observación \\
\hline 1 & $\begin{array}{l}\text { La ficha de registro cuenta con } \\
\text { suficiente información necesaria para } \\
\text { una posible investigación de carácter } \\
\text { forense. }\end{array}$ & & & \\
\hline 2 & $\begin{array}{l}\text { Existe claridad acerca de la utilidad de } \\
\text { la ficha de registro odontológico }\end{array}$ & & & \\
\hline 3 & $\begin{array}{l}\text { La ficha de registro tiene coherencia } \\
\text { con respecto al objetivo de su uso }\end{array}$ & & & \\
\hline 4 & $\begin{array}{l}\text { Cree usted que la ficha de registro } \\
\text { tenga la importancia y relevancia } \\
\text { necesaria. }\end{array}$ & & & \\
\hline 5 & $\begin{array}{l}\text { La ficha de registro tiene facilidad y } \\
\text { toma poco tiempo en el llenado de la } \\
\text { misma }\end{array}$ & & & \\
\hline 6 & $\begin{array}{l}\text { Cree usted que estos datos permitirán } \\
\text { tener ayuda en casos de investigación }\end{array}$ & & & \\
\hline
\end{tabular}

Observaciones generales:

Validado por:

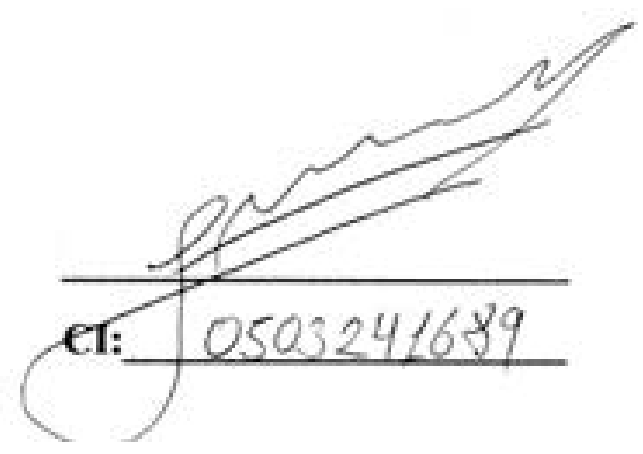

